\title{
A modelling study on discrimination tasks
}

\author{
Jianfeng Feng ${ }^{\mathrm{a}, \mathrm{b}, *}$, Feng Liu ${ }^{\mathrm{a}, \mathrm{c}}$ \\ ${ }^{a}$ COGS, University of Sussex, Brighton BN1 9QH, UK \\ ${ }^{\mathrm{b}}$ Newton Institute, Cambridge University, Cambridge CB3 OEH, UK \\ ${ }^{\mathrm{c}}$ Department of Physics, Nanjing University, Nanjing 210093, People's Republic of China
}

Accepted 22 August 2002

\begin{abstract}
We investigate the discrimination capacity of a single neuron model including the integrate-and-fire (IF) model and the IF-FHN model [Neural Networks, 14 (2001) 955], both theoretically and numerically. Both magnitude and frequency discrimination tasks are considered. It is found that for the magnitude discrimination task, with a small fraction of coherent inputs, an IF model and an IF-FHN model with inhibitory inputs can tell one input from the other. The total probability of misclassifications (TPM) is considerably reduced with the increasing of inhibitory inputs. However, the IF model and the IF-FHN model are incapable of performing the frequency discrimination task.

(C) 2002 Elsevier Science Ireland Ltd. All rights reserved.
\end{abstract}

Keywords: Neuron model; Integrate-and-fire model; IF-FHN model; Discrimination

\section{Introduction}

Recently, much attention has centred on the issue how the nervous system discriminates between input signals (Parker and Newsome, 1998; Romo and Salinas, 2001). To efficiently tell apart different input signals is of vital importance to the nervous system. However, the neural mechanisms underlying such activity remain one of the most significant and puzzling problems in neuroscience, despite there are mounting experimental and

\footnotetext{
* Corresponding author. http://www.cogs.susx.ac.uk/users/ jianfeng

E-mail addresses: jianfeng@cogs.susx.ac.uk (J. Feng), fliu@netra.nju.edu.cn (F. Liu).
}

theoretical results devoted to the topic (for example see recent reviews by Parker and Newsome, 1998; Romo and Salinas, 2001; Britten et al., 1992; Shadlen et al., 1996). Especially, Newsome and his colleagues have carried out a series of experiments during which monkeys are presented with displays of a set of moving dots. A fraction of the dots are moving coherently in one direction (either leftwards or rightwards), while the others are moving around randomly. The monkey is asked to make a quick eye movement in that coherent direction. Experimentally, it is found that the eye movements of the monkey can be predicted based upon the recorded pulse activity of individual MT neurons. Similar findings have also been reported for tactile, olfactory and aural stimuli (Romo and Salinas, 2001). These experiments are so intriguing in that 
they can directly demonstrate the link between neural activity and cognitive processes.

It is natural to ask if we can mimic the above experimental results via computational models of single neuron. In other words, can single model neuron accomplish a discrimination task with the similar accuracy as reported in experiments? Here we first propose a simple, but nevertheless informative, approach by modelling the moving direction of each dot by the rate of a Poisson process, which injects synaptic current to the neuron under study. Hence the input signal rate to the neuron is described by

$\frac{a[\cos (F t)+1]}{2}$

In the first place we use Poisson processes with a constant rate, i.e. in Eq. (1) $F=0$ and the model receives inputs with different values of $a$. Based on the firing rate histograms, the neuron can perfectly discriminate between two coherently moving directions as long as the dot coherence in display exceeds a critical value. The discrimination task is called magnitude discrimination task.

On the other hand, if the (visual) stimulus is represented by periodically modulated Poisson processes, i.e. in Eq. (1) $F$ is different for different input signals (for simplicity of notation we then fix $a$ ), the neuron can not efficiently perform the discrimination tasks. The discrimination task with this set up is termed frequency discrimination task and we refer the reader to Romo and Salinas (2001) for experimental reports on the similar set up in the somatosensory cortex.

Discriminating between different input signals is probably more fundamental constrains on the neural system than others such as maximising input-output information or redundancy reductions, as recently echoed in Barlow (2001). To understand, it will reveal principles employed by neuronal systems, which remain mysterious to us. The issue discussed here is currently a hot topic in neuroscience (for example see Kast, 2001). Our approach, as a first step to build models on decision-making and a complement to the experimental work, is still very rudimentary. However, we expect that our approach opens up many interesting questions to be further investigated in future (see Section 5).

Due to the space limit here, we only present the results for the integrate-and-fire (IF) model and the IF-FHN model, and refer the reader to our full papers (Feng and Liu, 2001; Liu and Feng, 2001) for the Hodgkin-Huxley (HH) model which behaves differently.

\section{Models}

We begin with the IF model (Brown et al., 1999; Feng, 1997; Tuckwell, 1988). When the membrane potential $V_{\mathrm{t}}$ is below the threshold $V_{\text {thre }}$, it is given by

$\mathrm{d} V_{\mathrm{t}}=-L\left(V_{\mathrm{t}}-V_{\text {rest }}\right) \mathrm{d} t+\mathrm{d} I_{\text {syn }}(t)$

where $L$ is the decay coefficient and the synaptic input is

$I_{\mathrm{syn}}(t)=w_{1} \sum_{i=1}^{p} E_{i}(t)-w_{2} \sum_{j=1}^{q} I_{j}(t)$

with $E_{i}(t), I_{i}(t)$ as Poisson processes with rate $\lambda_{i, E}$ and $\lambda_{i, I}$, respectively, $w_{1}>0, w_{2}>0$ are magnitude of each EPSP and IPSP. $p$ and $q$ are the total number of active excitatory and inhibitory synapses. Once $V_{\mathrm{t}}$ crosses $V_{\text {thre }}$ from below, a spike is generated and then $V_{\mathrm{t}}$ is reset to $V_{\text {rest }}$, the resting potential. The interspike interval of efferent spikes is

$T=\inf \left\{t: V_{\mathrm{t}} \geq V_{\text {thre }}\right\}$

For simplicity of notation we further assume that $q=p, \lambda_{i, I}=r \lambda_{i, E}=r \lambda_{i}$ for $i=1, \ldots, p$. Hence $r$ is the ratio between the inhibitory inputs and excitatory inputs.

To mimic the actual inputs to an MT neuron used in the experiments, we assume that $p_{c}$ out of $p$ dots move in an identical (coherent) direction, either leftwards or rightwards, and that other $p-p_{c}$ dots move randomly. As discussed in the Section 1 , the motion of each dot is coded by the firing rate of a single synaptic (Poisson) input. It is assumed that $\lambda_{i}=25$ or $75 \mathrm{~Hz}$ represents the input of leftand rightwards motion, respectively. For random movements, $\lambda_{i}$ is taken the value between 0 and 
$100 \mathrm{~Hz}$. This means that the coherence level in the input is $p_{c} / p$. In addition, we assume that a small amount of correlations are added to the synaptic inputs representing coherent moving dots (with the correlation coefficient $c=0.1$ ). The correlation considered here reflects the correlation of activity of different synapses (Feng, 2001; Zohary et al., 1994), rather than that of single incoming EPSP or IPSP. We refer the reader to Feng (2001) for a detailed discussion on the meaning of the correlation considered here. In other words, the input signal is masked by 'false' signal or noise. Our task is to test whether the neuron can detect the masked signal.

For the frequency discrimination task, our set up is even simple. The neuron receives unmasked signals, i.e. it receives $p$ Poisson synaptic inputs with a common rate $a[\cos (F t)+1] / 2$. Hence $p_{c}=p$. The discrimination task is to tell one input with frequency $F_{1}$ from the other with frequency $F_{2} \neq$ $F_{1}$, via efferent spike trains.

The IF model is the simplest neuron model which mimics certain properties of a biological neuron and is linear before resetting. A slightly more complex model is the IF-FHN model, an IF model but with a non-linear leakage coefficient, as in a biophysical model. In terms of the output signal-to-noise ratio, we know that the IF and IFFHN model behave in totally opposite ways when they receive correlated inputs (see Feng, 2001 for a review). We then naturally ask that whether the phenomenon observed for the IF model with partially correlated inputs is true only for the IF model or not. To this end we simulate the IF-FHN model, defied by

$$
\begin{aligned}
\frac{\mathrm{d} v(t)}{\mathrm{d} t}= & -\left(\frac{1}{\beta}+\gamma \alpha\right) v(t)+\gamma(1+\alpha) \frac{v(t)^{2}}{60}-\frac{\gamma v(t)^{3}}{3600} \\
& +\frac{\mathrm{d} I_{\mathrm{syn}}(t)}{\mathrm{d} t}
\end{aligned}
$$

when $v(t)$ (membrane potential) $<v_{\text {thre }}=50$. We can rewrite the IF-FHN model in the following form

$$
\begin{aligned}
\frac{\mathrm{d} v(t)}{\mathrm{d} t}= & -\left[\left(\frac{1}{\beta}+\bar{\gamma} \alpha\right)-\bar{\gamma}(1+\alpha) \frac{v(t)}{60}+\frac{\gamma v(t)^{2}}{3600}\right] v(t) \\
& +\frac{\mathrm{d} I_{\mathrm{syn}}(t)}{\mathrm{d} t}
\end{aligned}
$$

an IF model with a non-linear leakage term (Feng, 2001)

$\frac{1}{\beta}+\bar{\gamma} \alpha-\bar{\gamma}(1+\alpha) \frac{v(t)}{60}+\frac{\bar{\gamma} v(t)^{2}}{3600}$

The parameters are $v_{\text {rest }}=0, \gamma=50, \alpha=0.2, \beta=$ $2.5, w_{1}=w_{2}=2, p=300$. Note that to ensure the output firing rates in the similar regions for different models, the value of $p$ and $w_{1}$ used in the IF-FHN model is higher than that in the IF model (see next section), but still in the physiologically plausible regions.

\section{Magnitude discrimination tasks}

The parameters used in simulations of the IF model are $V_{\text {thre }}=20 \mathrm{mV}, V_{\text {rest }}=0 \mathrm{mV}, L=1 / 20$, $w_{1}=w_{2}=1 \mathrm{mV}, p=100$. In the perception experiments (Parker and Newsome, 1998), input patterns with the same dot coherence are repeatedly presented to stimulate the monkey to make a decision. Therefore, to explore the discrimination task we should take into account the firing rate histograms, rather than only the average firing rate. The firing rate of the neuron is estimated in a 10-s time window for one realisation. After that $\left\{\lambda_{i}\right.$, $\left.i=p_{c}+1, \ldots, p\right\}$ are redistributed, but the value of $p_{c} / p$ is fixed.

The firing rate histograms constructed from 100 different realisations are shown in Fig. 1, which depicts histograms of firing rates with purely excitatory $r=0$ (left column) and almost balanced excitatory and inhibitory inputs $r=0.95$ (right column) for $p_{c} / p=0.1$ (upper panel) and 0.25 (bottom panel), respectively. It is easily seen that when $p_{c} / p=0.25$ and $r=0.95$, from the observation of single-neuron activity, we could perfectly discriminate between leftward and rightward motions. Nevertheless, when $r=0$ and even $p_{c} / p=$ 0.25 we are still not able to perfectly tell leftwards from rightwards motions. 

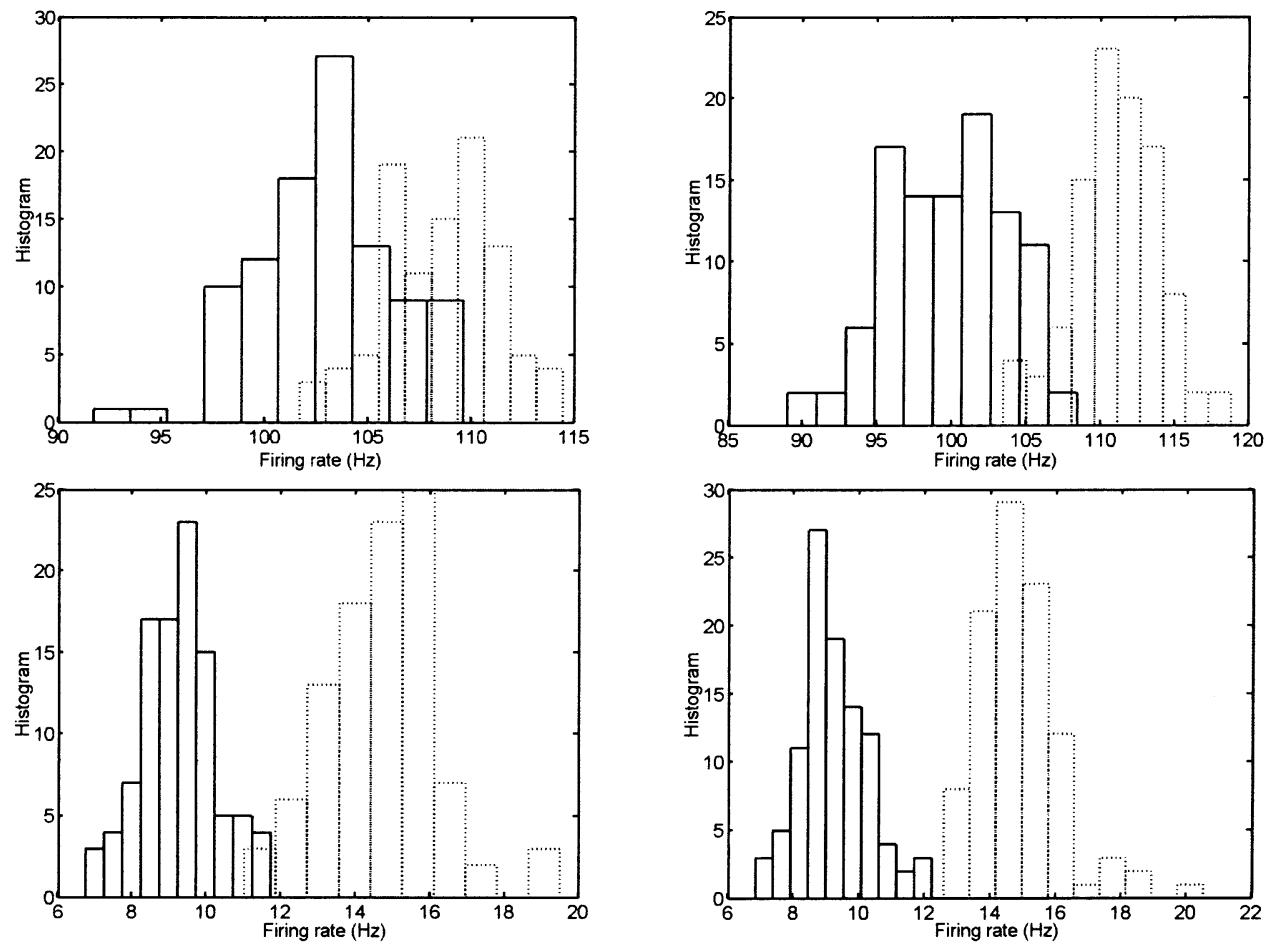

Fig. 1. Histogram of firing rates with $c=01.1$ for the IF model. Left, exclusively excitatory inputs $r=0$. Right, $r=0.95$. Upper panel: $p_{c}=10$. Bottom panel: $p_{c}=25$. It is easily seen that the two histograms move away from each other with the increasing of $r$. When $p_{c}=25, r=0.95, \mathrm{TPM}=0$.

To quantitatively characterise the discrimination capability of the neuron, we compute the minimal total probability of misclassification (TPM) defined by

$\mathrm{TPM}=\frac{1}{2} P$ (leftward|input is rightward $)$

$$
+\frac{1}{2} P \text { (rightward|input is leftward) }
$$

For example, in Fig. 1, we see that TPM for the left upper panel is about 16.77 and for the right upper panel is 2.4. Therefore, adding inhibitory inputs to the neuron considerably enhances its discrimination capability.

We can also prove theoretically that the firing rate histograms for two input patterns can be separated from each other after some value of $p_{c}$ l $p$, and that increasing inhibitory inputs to the model does make the histogram more widely separate thus enhancing the discrimination capability. The detailed results are presented in another publication (Feng and Liu, 2001). It is worth noting that based on the histogram of coefficient of variation (CV) of efferent spike trains, we also can tell apart two different coherent movements (results not shown here).

Fig. 2 shows the simulation results for the IFFHN model. It is readily seen that all conclusions for the IF model remain true: increasing inhibitory inputs considerably increases the discrimination capability of the model neuron. Furthermore, the fraction of coherent inputs which ensures a perfect discrimination is less than that of the IF model. For example, in Fig. 2, with $p_{c} / p=25 / 300$ of coherent inputs the histograms of efferent frequency are well separated when $r=1$. 

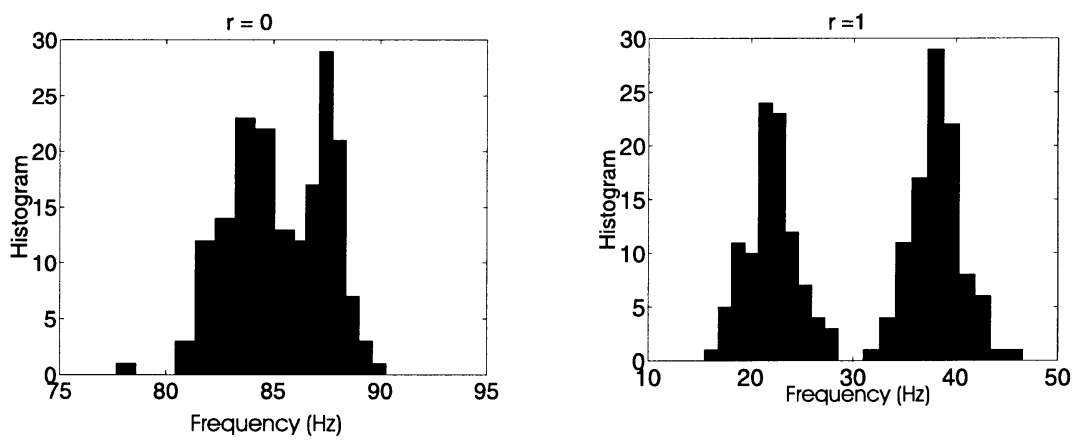

Fig. 2. Histogram of efferent frequency (Hz) of the IF-FHN model with $c=0.1, p_{c}=25, p=300$. Left, exclusively excitatory inputs $r=0$. Right, $r=1$.

\section{Frequency discrimination tasks}

It is noted that in the previous section, we model the visual stimulus by stationary Poisson inputs, which do not contain explicit information about time. Temporally changing Poisson input may be of more biological relevance (Romo and Salinas, 2001). Therefore, in the following we still represent the moving direction of each dot by the rate of Poisson process, but it is modulated by a periodic signal, i.e. $\lambda=a[\cos (2 \pi F t)] / 2$. Hence the moving direction is encoded by the modulation frequency.

Note that the set up of the frequency discrimination task is much simpler than the magnitude discrimination task. Here the input signal is not masked by 'false' signals or noise. As in Section 3, the issue we address here is: whether one could discriminate between two input patterns (two different $F$ ) from an observation of the efferent firing rate.

We plot the efferent firing rate and $\mathrm{CV}$ versus $F$ in Fig. 3 (upper panel). Apparently, for the IF model, the rate does not vary at all with $F$, only depending on the ratio of excitatory to inhibitory synapses. CV almost monotonically decreases with $F$ for $r=0$ and 0.6 , while it changes slightly for $r=1$. This implies that the CV histograms may be used to discriminate between different input frequencies. That is, the second-order or higher, statistics of outputs is needed to tell apart the modulation signal frequency. The observed phenomenon reflects the intrinsic mechanism of the IF model, it is linear before resetting per se. Fig. 3 (bottom panel) shows the firing interspike interval histograms. Clearly, the histograms mix together for different inputs.

Our conclusions are then tested for the IF-FHN model, for the IF model with reversal potentials, the IF model with a subthreshold oscillation. In Fig. 4 the numerical results for the IF-FHN model are shown. We see from Fig. 4 that the output frequency is flat for different model parameters. This confirms our main conclusion: a model with an integrate and fire mechanism is insensitive to the fluctuations in time domain, no matter whether the leakage is linear, non-linear (as in the IF-FHN model), or depending on incoming signals (as in the IF model with reversal potentials, not shown here).

As a matter of fact, we also explore the $\mathrm{HH}$ neuronal model, the model neuron can discriminate between the different modulation frequencies exclusively based on the firing rate histograms (results not shown here and see Liu and Feng, 2001). As we have discussed in Feng (2001), the IF model is largely different from the $\mathrm{HH}$ model in many ways.

\section{Discussion}

We have considered the problem of discriminating between input signals in terms of an observation of efferent spike trains of single neuron. It is 

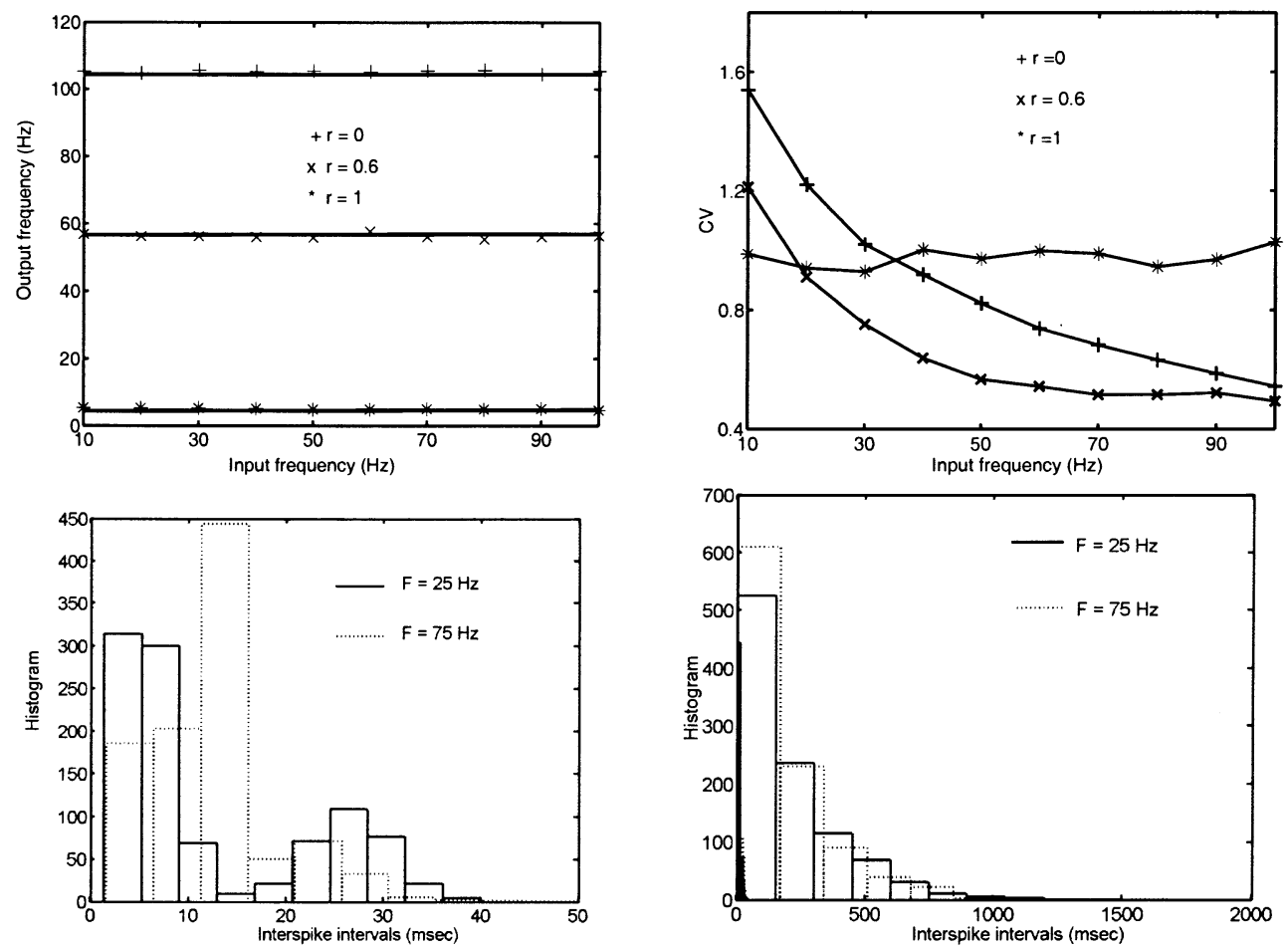

Fig. 3. Upper panel: mean firing time vs. frequency $F$ (left) and CV vs. frequency $F$ with $r=0,0.6,1$. Bottom panel: histograms of interspike intervals with different input temporal frequencies with $r=0.6$ (left) and $r=1$ (right). When $r=0.6$, a slowly vibrating stimulus results in a bimodal histogram $(F=25 \mathrm{~Hz}$ ); while a fast stimulus gives rise to a uni-modal distribution. When $r=1$, exactly balanced inputs, the efferent interspike intervals histograms are uni-modal and are similar to Poisson process.

found that the IF model is capable of performing magnitude discrimination tasks, roughly in agree- ment with experimental data, but is totally 'blind' on time domain.
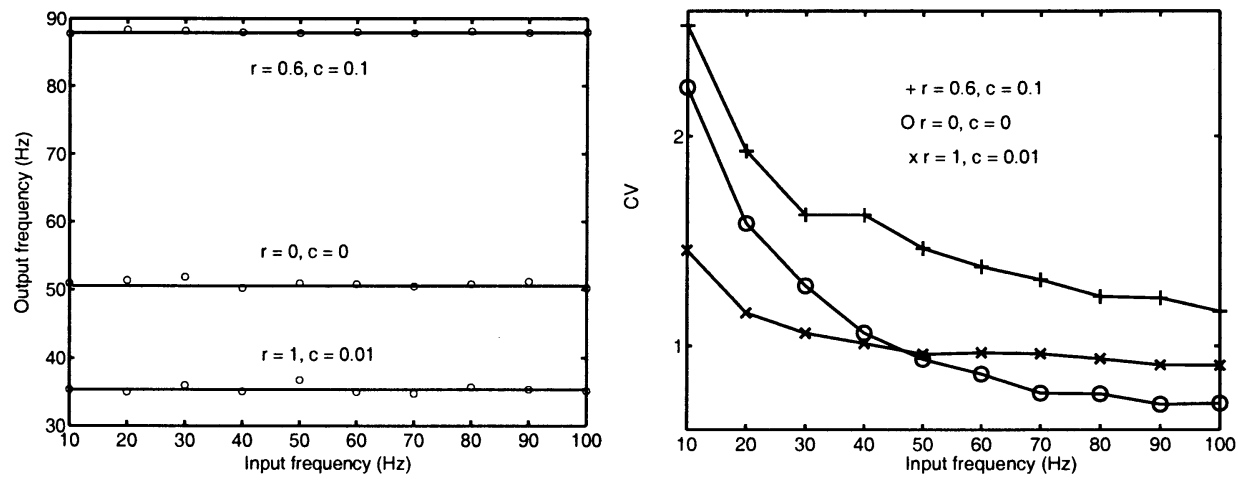

Fig. 4. Output frequency and CV vs. input temporal frequency for the IF-FHN model. 
There are many issues to be further explored in the future.

- We only considered the task of discrimination and have not included the time constrain. Definitely a neuronal system requires to tell one signal from the other within a time window as short as possible.

- The input signals used here are vary naive. To transform an image of moving dots to inputs specified in our paper requires a neural network to pre-process the input information. Hence to devise a network model (spiking neural networks or Reichardt detector, Borst, 2000) to test our results is one of our ongoing research topics.

- A neuronal system without learning is a 'dead' system. In actual situations, we all know that learning is prevailing in neuronal systems. Hence a reasonable learning rule should increase the discrimination capability of different input signals. There are several learning rules reported in the literature. To see the impact of them on discrimination task is an intriguing issue.

\section{Acknowledgements}

The work was in part supported by an EPSRC grant (GR/R54569), a grant of the Royal Society and an exchange grant of the Royal Society. F. Liu acknowledges the support from NNSF of China 30070208 .

\section{References}

Barlow, H., 2001. Redundancy reduction revisited. Network 12, 241-254.

Borst, A., 2000. Models of motion detection. Nat. Neurosci. 3, 1168.

Britten, K.H., Shadlen, M.N., Newsome, W.T., Celebrini, S., Movshon, J.A., 1992. The analysis of visual motion: a comparison of neuronal and psychophysical performance. J. Neurosci. 12, 4745-4765.

Brown, D., Feng, J.F., Feerick, S., 1999. Variability of firing of Hodgkin-Huxley and FitzHugh-Nagumo neurons with stochastic synaptic input. Phys. Rev. Lett. 82, 4731-4734.

Feng, J.F., 1997. Behaviours of spike output jitter in the integrate-and-fire model. Phys. Rev. Lett. 79, 4505-4508.

Feng, J.F., 2001. Is the integrate-and-fire model good enough - a review. Neural Networks 14, 955-975.

Feng, J.F., Liu, F., 2001. Discriminating between input signals via single neuron activity (submitted for publication).

Kast, B., 2001. Decisions, decisions .... Nature 411, 126-128.

Liu, F., Feng, J.F., 2001. Neuronal responses to stationary and dynamic Poisson synaptic inputs, submitted for publication.

Parker, A.J., Newsome, W.T., 1998. Sense and the single neuron: probing the physiology of perception. Annu. Rev. Neurosci. 21, 227-277.

Romo, R., Salinas, E., 2001. Touch and go: decision mechanisms in somatosensation. Annu. Rev. Neurosci. 24, 107137.

Shadlen, M.N., Newsome, W.T., 1996. Motion perception: seeing and deciding. Proc. Natl. Acad. Sci. 93, 628-633.

Tuckwell, H.C., 1988. Introduction to Theoretical Neurobiology, vol. 2. Cambridge University Press.

Zohary, E., Shadlen, M.N., Newsome, W.T., 1994. Correlated neuronal discharge rate and its implications for psychophysical performance. Nature 370, 140-143. 